\title{
Association of Visit-to-Visit Variability of Hemoglobin A1c and Medication Adherence
}

\author{
Ambili Ramachandran, MD, MS; Michael Winter, MPH; and Devin M. Mann, MD, MS
}

\begin{abstract}
BACKGROUND: Medication nonadherence is widespread, but there are few efficient means of detecting medication nonadherence at the point of care. Visit-to-visit variability in clinical biomarkers has shown inconsistent efficiency to predict medication adherence.

OBJECTIVE: To examine the performance of visit-to-visit variability (VVV) of hemoglobin A1c to predict nonadherence to antidiabetic medications.

METHODS: In this cross-sectional study using a clinical and administrative database, adult members of a managed care plan at a safety-net medical center from 2008 to 2012 were included if they had $\geq 3$ noninsulin antidiabetic prescription fills within the same class and $\geq 3$ A1c measurements between the first and last prescription fills. The independent variable was VVV of A1c (within-subject standard deviation of A1c), and the dependent variable was medication adherence (defined by medication possession ratio) determined from pharmacy claims. Unadjusted and adjusted multivariate logistic regression models were created to examine the relationship between VVV of A1c and medication nonadherence. Receiver-operating characteristic (ROC) curves assessed the performance of the adjusted model at discriminating adherence from nonadherence.
\end{abstract}

RESULTS: Among 632 eligible subjects, mean A1c was $7.7 \% \pm 1.3 \%$, and $83 \%$ of the sample was nonadherent to antidiabetic medications. Increasing quintiles of VVV of A1c and medication nonadherence were both associated with increased within-subject mean A1c and younger subject age. The logistic regression model (adjusted for age, sex, race/ethnicity, within-subject mean A1c, number of A1c measurements, number of days between the first and last antidiabetic medication prescription fills, and rate of primary care visits during the study period) showed a nonsignificant association of VVV of A1C and medication nonadherence ( $O R=1.19,95 \%$

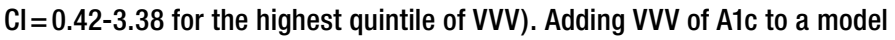
including age, sex, and race only modestly improved the C-statistic of the ROC curve from 0.6786 to 0.7064 .

CONCLUSIONS: VVV of A1c is not a robust predictor of antidiabetic medication nonadherence. Further innovation is needed to develop novel methods of detecting nonadherence.

J Manag Care Spec Pharm. 2015;21(3):229-37

Copyright $\odot 2015$, Academy of Managed Care Pharmacy. All rights reserved.

\section{What is already known about this subject}

Nonadherence to antidiabetic medications is common; however, existing methods of detecting nonadherence are time consuming, inefficient, or reliant on integrated pharmacy data not available to all providers.

Visit-to-visit variability (VVV) in clinical biomarkers has been shown to predict medication adherence in some medical conditions but has not been examined using biomarkers for diabetes such as hemoglobin Alc.

\section{What this study adds}

Using information from a clinical and administrative database, $83 \%$ of patients at an urban health center were found to be nonadherent to noninsulin antidiabetic medications, with adherence defined by a medication possession ratio of $80 \%$ or greater.

The proportion of subjects who were nonadherent to antidiabetic medications increased with increasing quintile of VVV of Alc, yet in the adjusted logistic regression model, there was no significant association between VVV of Alc and medication nonadherence. When added to other clinical variables, VVV of hemoglobin Alc is not a robust predictor of nonadherence to antidiabetic medications.

1 T onadherence to diabetic medications is a prevalent and costly problem. Patients take only an estimated 65\%$85 \%$ of prescribed doses of oral hypoglycemic agents. ${ }^{1,2}$ Lower rates of adherence are observed in racial minorities, ${ }^{3}$ Medicaid enrollees, ${ }^{1}$ and younger patients. ${ }^{4-6}$ Nonadherence to diabetic medications, antihypertensives, and cholesterollowering medications is associated with an increased risk of hospitalization and all-cause mortality, ${ }^{7}$ while satisfactory adherence is associated with decreased likelihood of microvascular complications. ${ }^{8}$ For every $10 \%$ increase in adherence to antidiabetic medications, total annual health care costs are projected to decrease by approximately $8.6 \% .{ }^{9,10}$

In order to achieve the benefits of adequate glycemic control, providers and health systems must be able to accurately detect nonadherence. Yet clinicians are insensitive appraisers of adherence, usually overestimating current and future adherence. ${ }^{11}$ Existing methods of assessing adherence, such as pill counts or validated questionnaires, are vulnerable to social desirability influences and consume time to complete. ${ }^{12,13}$ Unless they are working within an integrated pharmacy system, such as the Veterans Health Administration or health maintenance organizations, providers rarely have access to pharmacy refill information. Sophisticated techniques such as medication event monitoring systems (MEMS) are not widely available outside research studies and require integration of electronic information into the patient record during the relevant clinical visit. Thus, there is a need for efficient, reliable methods to diagnose nonadherence at the point of care. ${ }^{14}$

One candidate indicator of low medication adherence is variability in clinical biomarkers that are directly affected by medications. For example, a patient with hypertension 


\section{FIGURE 1 Study Flow Diagram}

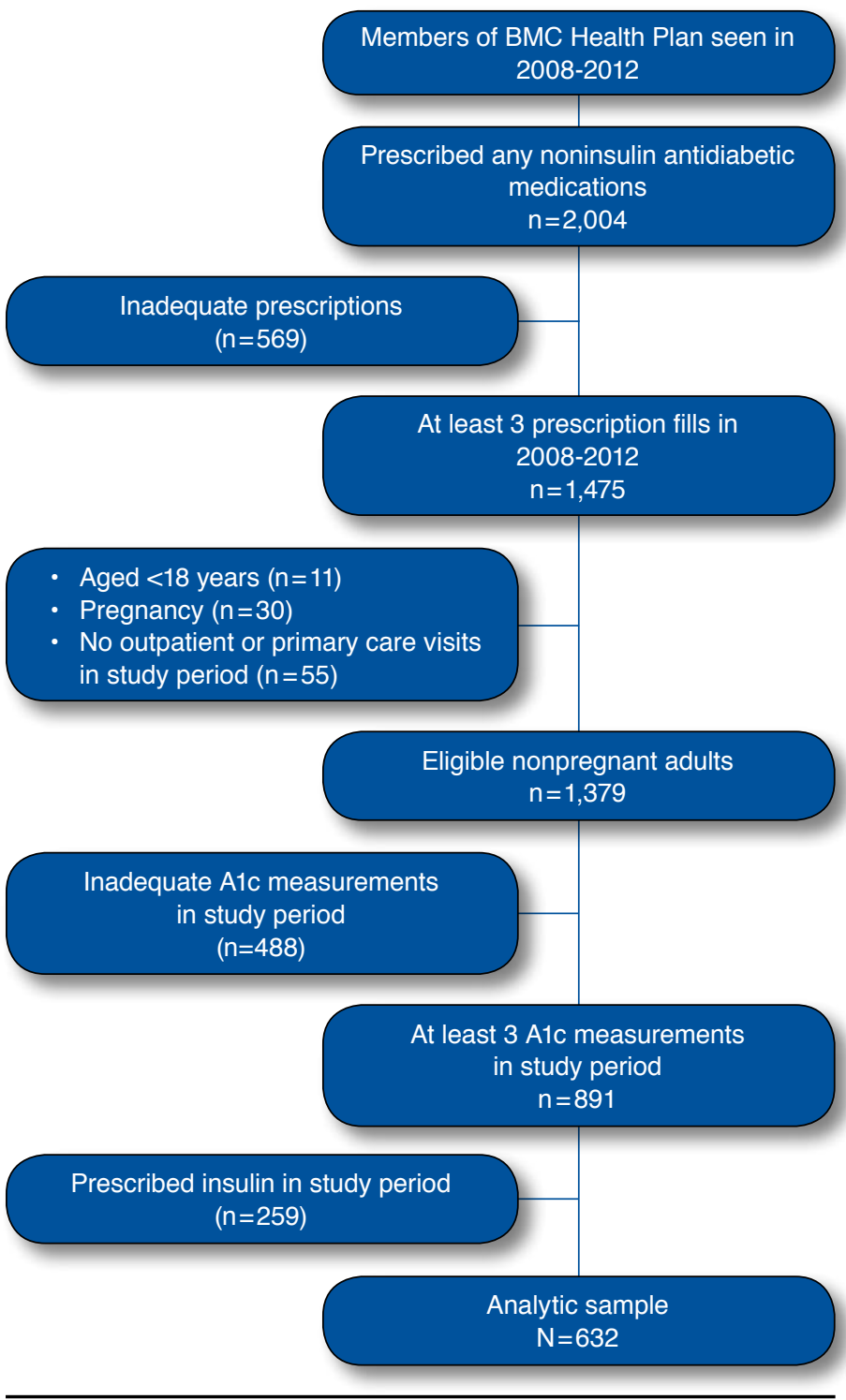

$A 1 c=$ hemoglobin $A 1 c ; B M C=$ Boston Medical Center

who is regularly taking antihypertensive medications would be expected to have relatively consistent blood pressure measurements over time. In contrast, inconsistent intake of antihypertensives would be associated with variable blood pressure recordings. In practice, such erratic readings often alert clinicians to possible poor adherence. Rather than rely on clinical intuition alone, this visit-to-visit variability (VVV) in the clinical biomarker could be quantified using electronic health record algorithms and used objectively as a predictor of medication nonadherence during the clinical visit.
Using pharmacy claims data, our group has previously demonstrated that VVV of low-density lipoprotein cholesterol (LDL-C) is associated with nonadherence to statin medications..$^{15}$ A similar analysis among patients with hypertension also showed a relationship between increasing VVV of systolic blood pressure and degree of antihypertensive medication nonadherence; however, medication adherence explained only a small fraction of VVV of blood pressure. ${ }^{16}$ There remains uncertainty about the ability of VVV of other biomarkers to predict nonadherence to medications.

Given the strong effect that antidiabetic medications have on glycosylated hemoglobin (Alc), we hypothesized that VVV of Alc would be a reliable predictor of nonadherence to antidiabetic medications. VVV of Alc could then be used in the clinical setting to identify patients with low adherence for targeted interventions to improve medication compliance.

\section{Methods}

\section{Data Source}

Our study sample consisted of patients enrolled in the Boston Medical Center (BMC) Health Plan who received care at BMC or 1 of 8 affiliated community health centers (CHCs) from 2008 to 2012. BMC is the largest safety-net hospital in New England, and the BMC Health Plan is a managed care organization that offers mostly Medicaid and free or heavily subsidized care.

BMC and its affiliated CHCs participate in the Massachusetts Healthcare Disparities Repository (MHDR), a collaborative program that promotes investigations in disparities in access to care and health outcomes by using existing clinical data. ${ }^{17}$ The MHDR employs the Informatics for Integrating Biology and the Bedside (I2B2) platform to aggregate de-identified clinical data for research purposes. Data available in MHDR and coordinated in I2B2 include visit dates, diagnoses, laboratory results, and medications, which originate from the electronic health record. Claims data from the BMC Health Plan, including filled prescriptions, are also available through I2B2. This study was approved by the Boston University Institutional Review Board.

\section{Inclusion Criteria}

Our sample of interest included adults aged 18 years or older who were taking at least 1 medication for diabetes and who had received at least 1 clinical service in 2008-2012 at BMC (Figure 1). All but 1 subject had a diagnosis of diabetes recorded during the study period (International Classification of Diseases, Ninth Revision, Clinical Modification [ICD-9-CM] code 250.x). Eligible medication classes during this time period were biguanides (metformin); sulfonylureas (glipizide, glimepiride, glyburide); thiazolidinediones (pioglitazone, rosiglitazone); meglitinides (nateglinide, repaglinide); alpha-glucosidase inhibitors (acarbose, miglitol); dipeptidyl peptidase-4 (DPP-4) inhibitors (sitagliptin, linagliptin); incretin mimetics (exenatide, liraglutide); 
or combination products (e.g., glipizide/metformin). Subjects taking insulin were excluded given the complexity of measuring adherence to insulin using claims data alone and since diabetic patients requiring insulin represent a different clinical population from patients on noninsulin regimens. Subjects were required to have at least 3 prescription fills within a given class of diabetic medications during the study period and at least $3 \mathrm{Alc}$ measurements between the first and last prescription fill dates.

We further required that subjects have at least 1 visit within a primary care specialty (general medicine, internal medicine, family medicine, women's health, or geriatrics) to reflect subjects whose diabetes is partly managed in a primary care setting rather than exclusively by specialists. Pregnant subjects were also excluded (ICD-9-CM codes 630 to 679.99 and 760 to 779.99) given different glycemic goals and permitted therapies during pregnancy.

\section{Independent Variable}

The independent variable was the VVV of Alc between the first and last medication fulfillment dates during the 4-year study period. The VVV of Alc was defined as the within-subject standard deviation (SD) of Alc during the study period and was divided into quintiles for the purposes of analysis. Alc measurements that were outside the 0.1 and 99.9 percentiles were top and bottom coded to those values.

\section{Dependent Variable}

The dependent variable was adherence to diabetic medication, defined by the medication possession ratio (MPR). The MPR is calculated as the sum of total days' supply of the medication from the first to the last prescription fill, divided by the total number of days in this period..$^{18}$ The MPR was calculated separately for each drug class for all diabetic medications prescribed for a subject using prescription medication claims data from I2B2. Then, an average MPR was calculated with equal weighting of each drug class. The average antidiabetic medication MPR was dichotomized as nonadherent and adherent according to the traditional standard of $<80 \%$ and $\geq 80 \%$, respectively. ${ }^{12}$

\section{Covariates}

Potential covariates were chosen based on previously reported associations with medication adherence $e^{4-6,9,10,12,15,16,19}$ and availability in the MHDR I2B2 system: age at first medication fill during the study period; sex; race/ethnicity (White, Black, Hispanic, or other); total number of ambulatory visits during the study period; total number of primary care visits during the study period; rate of primary care visits during the study period (calculated as number of primary care visits per 6 months); within-subject mean Alc during the study period; number of Alc measurements during the study period; number of days between the first and last diabetic medication prescription fills; medication drug class; number of drug classes; and the diagnoses of hypertension (ICD-9-CM, codes 401.0x to 405.0x), ischemic heart disease (codes 410.0x to 414.9x), cerebrovascular disease (codes 430.0x to 438.9x), and chronic kidney disease (codes 585.0x to 585.9).

\section{Statistical Analyses}

Descriptive data are reported as percentages for categorical variables and mean \pm SD for continuous variables. Bivariate associations between covariates and quintiles of VVV of Alc, and between covariates and diabetic medication adherence, were tested using the chi-square test for categorical variables and the Wilcoxon rank-sum test for continuous variables.

Unadjusted and adjusted multivariate logistic regression models were created to examine the relationship between VVV of Alc and diabetic medication nonadherence. The adjusted model included covariates that were statistically significantly associated with nonadherence based on bivariate associations and variables deemed important to predicting nonadherence according to literature review. Collinearity diagnostics indicated possible collinearity among 3 variables: rate of primary care visits during the study period, number of days between the first and last diabetic medication prescription fills, and number of Alc measurements. Separate adjusted models were thus created using only 2 of these 3 variables. A fully adjusted model including all important covariates (age, sex, race/ethnicity, within-subject mean Alc, number of Alc measurements, number of days between the first and last diabetic medication prescription fills, and rate of primary care visits during the study period) was then created. Parameter estimates were similar across all adjusted models; therefore, the fully adjusted model was accepted as the final model.

Using the average MPR across drug classes, a patient could be considered adherent if highly adherent to 1 drug class and less adherent to another drug class (e.g., MPR 88\% for metformin but MPR 74\% to sulfonylureas, yielding an average MPR of $82 \%)$. A stricter definition of adherence would require that the MPR be $\geq 80 \%$ for all drug classes. A sensitivity analysis of the fully adjusted model using this latter definition of adherence was performed.

The Hosmer-Lemeshow test for goodness-of-fit was used to evaluate the models. For all logistic regression models, the odds ratio (OR) and 95\% confidence intervals (CIs) were calculated, and $P<0.05$ was used for all significance levels. The performance of each model at discriminating diabetic medication adherence from nonadherence was assessed by plotting the receiver operator characteristic (ROC) curve and calculating the C-statistic. All analyses were conducted using SAS version 9.2 (SAS Institute, Cary, NC). 
Association of Visit-to-Visit Variability of Hemoglobin A1c and Medication Adherence

TABLE 1 Association of VVV of Alc and Sociodemographic and Clinical Variables, 2008-2012

VVV of Alc (Quintiles)

\begin{tabular}{|c|c|c|c|c|c|c|c|}
\hline & \multicolumn{6}{|c|}{ VVV of Alc (Quintiles) ${ }^{a}$} & \multirow[b]{2}{*}{$P$ Value } \\
\hline & $\begin{array}{c}\text { Q1: } \leq 0.31 \\
\mathrm{~N}=127, \mathrm{~N}(\%)\end{array}$ & $\begin{array}{c}\mathrm{Q} 2:>0.31-\leq 0.49 \\
\mathrm{~N}=125, \mathrm{~N}(\%)\end{array}$ & $\begin{array}{c}\text { Q3: }>0.49-\leq 0.75 \\
N=126, N(\%)\end{array}$ & $\begin{array}{c}\mathrm{Q} 4:>0.75-\leq 1.15 \\
\mathrm{~N}=128, \mathrm{~N}(\%)\end{array}$ & $\begin{array}{c}\text { Q5: > } 1.15 \\
\mathrm{~N}=126, \mathrm{~N}(\%)\end{array}$ & $\begin{array}{c}\text { Overall } \\
\mathrm{N}=632, \mathrm{~N}(\%)\end{array}$ & \\
\hline \multicolumn{8}{|l|}{ Sex } \\
\hline Female & $80.0 \quad(63.0)$ & $73.0 \quad(58.4)$ & $(51.6)$ & $69.0 \quad(53.9)$ & $58.0 \quad(46.0)$ & $345.0 \quad(54.6)$ & \multirow{2}{*}{0.0733} \\
\hline Male & $47.0 \quad(37.0)$ & $52.0 \quad(41.6)$ & $61.0 \quad(48.4)$ & $59.0 \quad(46.1)$ & $68.0 \quad(54.0)$ & $287.0 \quad(45.4)$ & \\
\hline Age at first Rx fill (years) & $\begin{array}{ll}53.8 & (8.3) \\
\end{array}$ & $52.1 \quad(8.0)$ & $52.8 \quad(7.9)$ & $50.5 \quad(7.7)$ & $49.1 \quad(9.3)$ & $51.7 \quad(8.4)$ & $<0.0001$ \\
\hline \multicolumn{8}{|l|}{ Race } \\
\hline White & $14.0 \quad(11.3)$ & $18.0 \quad(14.8)$ & $16.0 \quad(12.7)$ & $17.0 \quad(13.4)$ & $16.0 \quad(12.9)$ & $81.0 \quad(13.0)$ & \multirow{4}{*}{0.5383} \\
\hline Black & $66.0 \quad(53.2)$ & $61.0 \quad(50.0)$ & $60.0 \quad(47.6)$ & $61.0 \quad(48.0)$ & $75.0 \quad(60.5)$ & $323.0 \quad(51.9)$ & \\
\hline Hispanic & $24.0 \quad(19.4)$ & $16.0(13.1)$ & $19.0 \quad(15.1)$ & $22.0 \quad(17.3)$ & $16.0 \quad(12.9)$ & $97.0 \quad(15.5)$ & \\
\hline Other & $20.0(16.1)$ & $27.0 \quad(22.1)$ & $31.0 \quad(24.6)$ & $27.0 \quad(21.3)$ & $17.0 \quad(13.7)$ & $122.0 \quad(19.6)$ & \\
\hline Outpatient visits & $37.2(29.9)$ & $49.4 \quad(40.4)$ & $37.4 \quad(26.5)$ & $45.4 \quad(35.6)$ & $36.6 \quad(25.8)$ & $41.2 \quad(32.5)$ & 0.0143 \\
\hline Primary care visits ${ }^{\mathrm{b}}$ & $12.0 \quad(9.0)$ & $16.0 \quad(14.2)$ & $13.7 \quad(8.6)$ & $16.6 \quad(10.9)$ & $15.0 \quad(8.6)$ & $14.7 \quad(10.6)$ & 0.0003 \\
\hline Rate of primary care visits/6 months & $(3.0)$ & $3.1 \quad(4.9)$ & $(1.8)$ & $2.6 \quad(1.4)$ & $(1.5)$ & $2.8 \quad(2.8)$ & 0.8597 \\
\hline \multicolumn{8}{|l|}{ Diagnoses } \\
\hline Diabetes mellitus & $126.0 \quad(99.2)$ & $125.0(100.0)$ & $126.0(100.0)$ & $128.0(100.0)$ & $126.0(100.0)$ & $631.0 \quad(99.8)$ & 0.7975 \\
\hline Hypertension & $110.0 \quad(86.6)$ & $111.0 \quad(88.8)$ & $110.0 \quad(87.3)$ & $110.0 \quad(85.9)$ & $106.0 \quad(84.1)$ & $547.0 \quad(86.6)$ & 0.8644 \\
\hline Ischemic heart disease & $13.0 \quad(10.2)$ & $14.0 \quad(11.2)$ & $(9.5)$ & $12.0 \quad(9.4)$ & $16.0(12.7)$ & $67.0 \quad(10.6)$ & 0.9087 \\
\hline Cerebrovascular disease & $6.0 \quad(4.7)$ & $9.0 \quad(7.2)$ & (1.6) & $(5.5)$ & $5.0 \quad(4.0)$ & $29.0 \quad(4.6)$ & 0.2996 \\
\hline Chronic kidney disease & $13.0 \quad(10.2)$ & $(7.2)$ & $(7.9)$ & $(9.4)$ & $(7.9)$ & $(8.5)$ & 0.9118 \\
\hline Alc measurements & $4.9 \quad(2.5)$ & (3.3) & $(3.4)$ & $(3.7)$ & $(2.9)$ & (3.3) & $<0.0001$ \\
\hline Within-subject mean Alc & $\begin{array}{ll}6.7 & (0.9) \\
\end{array}$ & $6.9 \quad(0.8)$ & $\begin{array}{ll}7.7 & (0.9) \\
\end{array}$ & $8.1 \quad(1.2)$ & $8.9 \quad(1.2)$ & $7.7 \quad(1.3)$ & $<0.0001$ \\
\hline Days between first and last fill dates & $850.2(465.3)$ & $1,107.0(496.9)$ & $1,040.5(506.1)$ & $1,155.4(494.4)$ & $1,181.3(492.0)$ & $1,066.8(503.7)$ & $<0.0001$ \\
\hline \multicolumn{8}{|l|}{ Number of drug classes } \\
\hline 1 & $88.0 \quad(69.3)$ & $64.0 \quad(51.2)$ & $36.0 \quad(28.6)$ & $40.0 \quad(31.3)$ & $38.0 \quad(30.2)$ & $266.0 \quad(42.1)$ & \multirow{4}{*}{$<0.0001$} \\
\hline 2 & $29.0(22.8)$ & $47.0 \quad(37.6)$ & $65.0 \quad(51.6)$ & $54.0 \quad(42.2)$ & $66.0 \quad(52.4)$ & $261.0 \quad(41.3)$ & \\
\hline 3 & $10.0 \quad(7.9)$ & $14.0 \quad(11.2)$ & $23.0 \quad(18.3)$ & $32.0 \quad(25.0)$ & $22.0 \quad(17.5)$ & $101.0 \quad(16.0)$ & \\
\hline 4 & 0 & 0 & $2.0 \quad(1.6)$ & $2.0 \quad(1.6)$ & 0 & $4.0 \quad(0.6)$ & \\
\hline \multicolumn{8}{|l|}{ Antidiabetic MPR ${ }^{\mathrm{c}}$} \\
\hline$<80 \%$ & $101.0 \quad(79.5)$ & $96.0 \quad(76.8)$ & $101.0 \quad(80.2)$ & $110.0 \quad(85.9)$ & $118.0 \quad(93.7)$ & $526.0 \quad(83.2)$ & \multirow{2}{*}{0.0027} \\
\hline$\geq 80 \%$ & $26.0 \quad(20.5)$ & $29.0 \quad(23.2)$ & $25.0 \quad(19.8)$ & $18.0(14.1)$ & $8.0 \quad(6.4)$ & $106.0 \quad(16.8)$ & \\
\hline \multicolumn{8}{|c|}{$\begin{array}{l}\text { Note: Data are presented as mean } \pm S D \text { or } n(\%) \text {. } \\
\text { aVVV of Alc was defined by within-subject standard deviation of Alc. } \\
\text { bPrimary care visits were defined as visits to Family Medicine, General Medicine, Women's Health, Primary Care, and Geriatrics. } \\
{ }^{2} M P R \text { was averaged across medication classes for subjects taking medications from more than } 1 \text { drug class. Nonadherence was defined as MPR }<80 \% \text {. } \\
\text { Alc = hemoglobin Alc; MPR = medication possession ratio; } Q=\text { quintiles; } R x=\text { prescription; } S D=\text { standard deviation; } V V V=\text { visit-to-visit variability. }\end{array}$} \\
\hline
\end{tabular}

\section{Results}

A total of 632 adults with at least 3 noninsulin diabetic medication fills and at least $3 \mathrm{Alc}$ measurements between the first and last medication fills were identified between 2008 and 2012 (Figure 1). The majority of subjects were female (54.6\%) and Black (51.9\%), and the mean age in the sample was 52 years (Table 1). Subjects contributed an average of almost 3 years of pharmacy prescription information (mean 1,067 days [SD 504] between first and last prescription fills). Almost $58 \%$ of the sample was taking more than 1 drug for diabetes, most commonly metformin (89.9\%) and sulfonylureas (57.9\%; data not shown). The average within-subject mean Alc level was $7.7 \% \pm 1.3 \%$.

Table 1 shows the association between VVV of Alc and sociodemographic and clinical covariates. As the VVV of Alc increased, mean age decreased significantly. The number of Alc measurements, the within-subject mean Alc, and the number of days between the first and last medication fills increased significantly with increasing quintile of VVV of Alc. There was no significant association between VVV of Alc and race, sex, or proportion with comorbidities of interest.

Approximately $83 \%$ of the sample met criteria for nonadherence, and nonadherence was significantly associated with younger age and Black race (Table 2). A greater number of Alc measurements, higher within-subject mean Alc, and a longer interval between first and last fill dates were significantly associated with medication nonadherence. Subjects with hypertension were more likely to be adherent than those without hypertension; no significant associations were found for other diagnoses. 


\begin{tabular}{|c|c|c|c|c|c|c|c|}
\hline & \multicolumn{6}{|c|}{ Antidiabetic MPR ${ }^{a}$} & \multirow[b]{2}{*}{$P$ Value } \\
\hline & \multicolumn{2}{|c|}{$\begin{array}{c}\mathrm{MPR}<80 \% \\
\mathrm{~N}=526, \mathrm{~N}(\%)\end{array}$} & \multicolumn{2}{|c|}{$\begin{array}{c}\mathrm{MPR} \geq 80 \% \\
\mathrm{~N}=106, \mathrm{~N}(\%)\end{array}$} & \multicolumn{2}{|c|}{$\begin{array}{c}\text { Overall } \\
\mathrm{N}=632, \mathrm{~N}(\%)\end{array}$} & \\
\hline \multicolumn{8}{|l|}{$\underline{\operatorname{Sex}}$} \\
\hline Female & 287.0 & $(54.6)$ & 58.0 & $(54.7)$ & 345.0 & $(54.6)$ & \multirow{2}{*}{0.9768} \\
\hline Male & 239.0 & $(45.4)$ & 48.0 & $(45.3)$ & 287.0 & $(45.4)$ & \\
\hline Age at first Rx fill (years) & 51.0 & $(8.5)$ & 55.2 & $(6.5)$ & 51.7 & $(8.4)$ & $<0.0001$ \\
\hline \multicolumn{8}{|l|}{ Race } \\
\hline White & 63.0 & $(12.1)$ & 18.0 & $(17.3)$ & 81.0 & $(13.0)$ & \multirow{4}{*}{0.0099} \\
\hline Black & 284.0 & $(54.7)$ & 39.0 & $(37.5)$ & 323.0 & $(51.9)$ & \\
\hline Hispanic & 79.0 & $(15.2)$ & 18.0 & $(17.3)$ & 97.0 & $(15.5)$ & \\
\hline Other & 93.0 & $(17.9)$ & 29.0 & $(27.9)$ & 122.0 & $(19.6)$ & \\
\hline Outpatient visits & 42.0 & $(32.2)$ & 37.5 & $(33.8)$ & 41.2 & $(32.5)$ & 0.0490 \\
\hline Primary care visits ${ }^{\mathrm{b}}$ & 15.1 & $(9.7)$ & 12.6 & $(14.1)$ & 14.7 & $(10.6)$ & $<0.0001$ \\
\hline Rate of primary care visits/ 6 months & 2.7 & $(1.9)$ & 3.2 & $(5.5)$ & 2.8 & $(2.8)$ & 0.7451 \\
\hline \multicolumn{8}{|l|}{ Diagnoses } \\
\hline Diabetes mellitus & 525.0 & $(99.8)$ & 106.0 & $(100.0)$ & 631.0 & $(99.8)$ & 1.0000 \\
\hline Hypertension & 448.0 & $(85.2)$ & 99.0 & (93.4) & 547.0 & $(86.6)$ & 0.0277 \\
\hline Ischemic heart disease & 53.0 & $(10.1)$ & 14.0 & $(13.2)$ & 67.0 & $(10.6)$ & 0.3860 \\
\hline Cerebrovascular disease & 24.0 & $(4.6)$ & 5.0 & $(4.7)$ & 29.0 & $(4.6)$ & 1.0000 \\
\hline Chronic kidney disease & 43.0 & $(8.2)$ & 11.0 & $(10.4)$ & 54.0 & $(8.5)$ & 0.4593 \\
\hline Number of Alc measurements & 6.4 & $(3.2)$ & 5.8 & $(3.3)$ & 6.3 & $(3.3)$ & 0.0116 \\
\hline Within-subject mean Alc & 7.8 & $(1.3)$ & 7.2 & $(0.9)$ & 7.7 & $(1.3)$ & 0.0003 \\
\hline Days between first and last fill dates & $1,109.4$ & $(496.8)$ & 855.1 & $(486.3)$ & $1,066.8$ & $(503.7)$ & $<0.0001$ \\
\hline \multicolumn{8}{|l|}{ Number of drug classes } \\
\hline 1 & 216.0 & $(41.1)$ & 50.0 & $(47.2)$ & 266.0 & $(42.1)$ & \multirow{4}{*}{0.5150} \\
\hline 2 & 219.0 & $(41.6)$ & 42.0 & $(39.6)$ & 261.0 & $(41.3)$ & \\
\hline 3 & 87.0 & $(16.5)$ & 14.0 & $(13.2)$ & 101.0 & $(16.0)$ & \\
\hline 4 & 4.0 & $(0.8)$ & 0 & & 4.0 & $(0.6)$ & \\
\hline
\end{tabular}

Note: Data are presented as mean \pm SD or $n(\%)$.

${ }^{a}$ MPR was averaged across medication classes for subjects taking medications from more than 1 drug class. Nonadherence was defined as MPR $<80 \%$.

bPrimary care visits were defined as visits to Family Medicine, General Medicine, Women's Health, Primary Care, and Geriatrics.

$A l c=$ hemoglobin $A l c ; M P R=$ medication possession ratio; $R x=$ prescription $S D=$ standard deviation.

The proportion of subjects who were nonadherent increased overall with increasing quintiles of VVV of Alc $(79.5 \%, 76.8 \%$, $80.2 \%, 85.9 \%, 93.7 \%, P=0.0027$ for association; Table 1). In the unadjusted logistic regression model, there was a moderately strong association between VVV of Alc and nonadherence to diabetic medication that was statistically significant for the highest quintile (OR=3.80, 95\% CI=1.65-8.76; Table 3). However, the fully adjusted model showed no association between VVV of Alc and nonadherence. In a sensitivity analysis using the stricter definition of adherence (i.e., MPR $\geq 80 \%$ for all drug classes), there remained no significant association.

ROC curves for the models demonstrate that addition of VVV of Alc does not add substantially to the discrimination between adherence and nonadherence to diabetic medications (Figure 2). The C-statistic for the model when only age, sex, and race are included is 0.6786; adding VVV of Alc modestly improves the C-statistic to 0.7064 . Similarly, adding VVV of Alc to a model that includes age, sex, race, within- subject mean Alc, number of Alc measurements, number of days between first and last prescription fill dates, and rate of primary care visits improves the C-statistic marginally from 0.7460 to 0.7525 .

\section{Discussion}

Unlike previous studies examining the ability of VVV to predict medication nonadherence, ${ }^{15,16}$ we did not find that VVV of Alc was a significant predictor of nonadherence to noninsulin diabetic medications when added to other clinical information. However, consistent with prior research, ${ }^{20,21}$ younger age, Black race, and higher mean Alc levels were statistically significantly associated with medication nonadherence.

Given that nearly $60 \%$ of subjects were on more than 1 antidiabetic agent, polypharmacy may have complicated the relationship between medication adherence and VVV of Alc. Full therapeutic doses of metformin and thiazolidinediones can lower Alc by $1 \%-2 \%$ and $0.5 \%-1.4 \%$, respectively. ${ }^{22}$ In 
TABLE 3 Logistic Regression Models Predicting Odds of Antidiabetic Nonadherence According to Quintile of VVV of Alc, 2008-2012

\begin{tabular}{|c|c|c|c|c|}
\hline & \multicolumn{2}{|c|}{ Unadjusted Modela $^{a}$} & \multirow{2}{*}{$\begin{array}{c}\text { Final Adjusted Modelb } \\
\text { OR }(95 \% \text { CI })\end{array}$} & \multirow[b]{2}{*}{$P$ Value } \\
\hline & OR $(95 \% \mathrm{CI})$ & \multirow[t]{2}{*}{$P$ Value } & & \\
\hline \multicolumn{4}{|l|}{ VVV of Alcc } & \\
\hline lst quintile (reference) & 1.00 & \multirow{5}{*}{0.0052} & 1.00 & \multirow{5}{*}{0.4070} \\
\hline 2nd quintile & $0.85 \quad(0.47-1.55)$ & & $(0.32-1.21)$ & \\
\hline 3rd quintile & $1.04 \quad(0.56-1.92)$ & & $(0.32-1.33)$ & \\
\hline 4th quintile & $1.57 \quad(0.81-3.04)$ & & $(0.31-1.57)$ & \\
\hline 5th quintile & $3.80 \quad(1.65-8.76)$ & & $(0.42-3.38)$ & \\
\hline \multicolumn{5}{|l|}{ Sex } \\
\hline Female (reference) & & & 1.00 & \multirow{2}{*}{0.9133} \\
\hline Male & & & $(0.65-1.63)$ & \\
\hline \multicolumn{5}{|l|}{ Age at first antidiabetic Rx fill } \\
\hline 1-year increase & & & $(0.91-0.97)$ & 0.0004 \\
\hline \multicolumn{5}{|l|}{ Race } \\
\hline White & & & $(0.25-0.95)$ & \multirow{4}{*}{0.0371} \\
\hline Black (reference) & & & 1.00 & \\
\hline Hispanic & & & $(0.31-1.14)$ & \\
\hline Other & & & $(0.26-0.84)$ & \\
\hline Rate of primary care visits/6 months ${ }^{\mathrm{d}}$ & & & $(0.95-1.08)$ & 0.6937 \\
\hline Days between first and last fill dates & & & $(1.00-1.00)$ & $<0.0001$ \\
\hline Number of Alc measurements & & & $(0.78-0.98)$ & 0.0181 \\
\hline Within-subject mean Alc & & & $(1.06-1.89)$ & 0.0181 \\
\hline
\end{tabular}

Note: Nonadherence was defined as MPR $<80 \%$ averaged across antidiabetic medication classes.

${ }^{a}$ Hosmer-Lemeshow goodness-of-fit test, $P=1.000$

${ }^{b}$ Hosmer-Lemeshow goodness-of-fit test, $P=0.1734$.

cVVV of Alc was defined as within-subject standard deviation of Alc during the study period.

dPrimary care visits were defined as visits to Family Medicine, General Medicine, Women's Health, Primary Care, and Geriatrics.

$A l c=$ hemoglobin $A 1 c ; C I=$ confidence interval; $M P R=$ medication possession ratio; OR=odds ratio; $R x=$ prescription; $V V V=$ visit-to-visit variability.

comparison, the starting dose of simvastatin lowers LDL-C by $30 \% .{ }^{23}$ The stronger relationship between VVV of LDL-C and statin nonadherence ${ }^{15}$ may be due to use of a single agent to affect LDL-C and the powerful impact that statins have on cholesterol metabolism. The degree to which a medication can change a biomarker may affect the degree to which variability in the biomarker might be explained by medication adherence.

By reducing absolute Alc and fluctuations in glucose levels, improvements in diet, exercise, and weight may be expected to reduce Alc variability as well..$^{24}$ Unfortunately, in this study, weight data were available within 30 days of the first and last prescription fills for less than one-third of subjects. In the case of LDL-C, statin medications are even more potent than diet and exercise in altering cholesterol levels; therefore, adherence to statins may account for more variability in LDL than adherence to diabetic medications accounts for variability in Alc.

Finally, the natural history of diabetes is one of progressive insulin resistance and loss of endogenous insulin production leading to worsening glycemic control. Increase in Alc, and perhaps Alc variability as well, may be expected even with ideal medication adherence, although the rapidity may be altered by medication compliance and intensification. ${ }^{24,25}$

Many studies have shown lower rates of adherence among Black patients with diabetes, ${ }^{5,6,19}$ although this association is not consistent across diseases. ${ }^{12}$ Over half of our sample was Black, and while race was not associated with quintile of VVV of Alc, it was significantly associated with nonadherence. Alc levels are higher in Black individuals compared with White individuals across the glycemic spectrum, ${ }^{26}$ even when controlling for other predictors of Alc, and this discrepancy is most pronounced among diabetics. ${ }^{27}$ These racial differences should not affect VVV, which is a measure of within-subject variability, but it may explain differences in mean Alc by race in our sample. Our findings of lower adherence among younger subjects is consistent with other studies of medication adherence among diabetic patients, both young adults and seniors. ${ }^{4-6}$ This association may be related to duration of disease, severity of illness, or management of multiple chronic diseases. These demographic findings support interventions to improve adherence to diabetic medications among young patients and those from minority communities. 


\section{FIGURE 2 ROC Curves for Identification of} Antidiabetic Medication Nonadherence

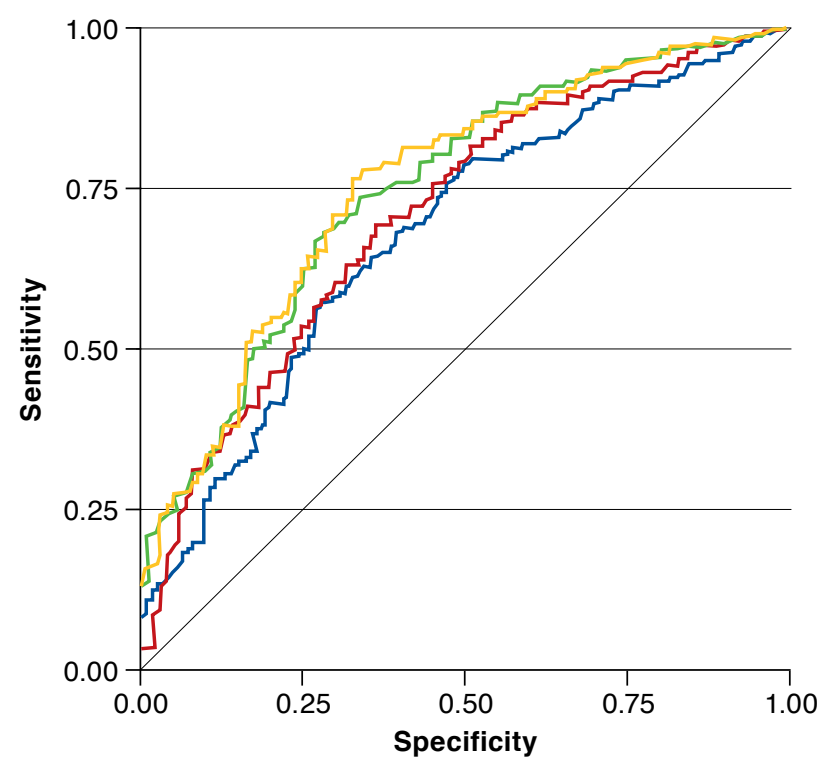

\begin{tabular}{l|l|c}
\hline Legend & \multicolumn{1}{|c}{ ROC Model } & $\begin{array}{c}\text { C-statistic } \\
\text { (Area Under Curve) }\end{array}$ \\
\hline \hline & Age, sex, race & 0.6786 \\
\hline & Age, sex, race, VVV of Alc & 0.7064 \\
\hline & $\begin{array}{l}\text { Age, sex, race, within-subject mean } \\
\text { Alc, number of Alc measurements, } \\
\text { number of days between first and } \\
\text { last prescription fill dates, rate of } \\
\text { primary care visits }\end{array}$ & 0.7460 \\
\hline & $\begin{array}{l}\text { Age, sex, race, within-subject mean } \\
\text { Alc, number of Alc measurements, } \\
\text { number of days between first and } \\
\text { last prescription fill dates, rate of } \\
\text { primary care visits, VVV of Alc }\end{array}$ & 0.7525 \\
\hline
\end{tabular}

Note: Sequential ROC curves for identification of antidiabetic nonadherence (defined as antidiabetic MPR $<80 \%$ ) using sociodemographic and clinical variables. Addition of VVV of Alc to the final model improves the C-statistic from 0.7460 to 0.7525 (95\% CI=0.7021-0.8029).

$A l c=$ hemoglobin $A 1 c ; C I=$ confidence interval; $M P R=$ medication possession ratio; $R O C=$ receiver operator characteristic; $V V V=$ visit-to-visit variability.

While we are the first to explore an association between VVV of Alc and medication adherence, increased variability in Alc is already recognized as a marker of elevated risk for diabetic complications. In the Finnish Diabetes Nephropathy longitudinal cohort study of patients with type 1 diabetes, investigators found that variability in Alc predicted development and progression of renal disease as well as cardiovascular events such as myocardial infarction and stroke. ${ }^{28}$ Similarly, among subjects with type 2 diabetes, researchers have found that variability in Alc predicted microalbuminuria and progression of nephropathy, independent of mean Alc. ${ }^{29,30}$ In a study using a clinical database, type 2 diabetics with high variability in Alc had nearly twice the risk of all-cause mortality compared with subjects with low variability. ${ }^{31}$ These findings parallel studies of variability in systolic blood pressure, which has been associated with increased risk for stroke and all-cause mortality, independent of mean blood pressure. ${ }^{32,33}$ More research is needed on the biological basis for this variability in clinical biomarkers and the extent to which specific medication classes and medication adherence may account for this variability.

\section{Limitations}

Our sample consists of insured but low-income patients who are largely of minority race/ethnicity at a single urban health care system; thus, the findings may not apply to other settings. Still, the challenge of medication nonadherence may be greatest in such a population and merits particular attention. Indeed, the MHDR database used in this study was intended to promote research in health care disparities according to race and socioeconomic status. As a younger, non-Medicare sample, this population is also where early, aggressive management of diabetes is essential.

Adherence to oral diabetic medications in most studies has ranged from $65 \%-85 \%$ yet is often lower (36\%-53\%) in populations that are similar to ours. ${ }^{1}$ The average MPR in our sample was $56.2 \%$, and the rate of medication nonadherence was $83.2 \%$, again limiting generalizability but arguing for the disproportionate burden of the problem of adherence in this community.

Over half of our subjects had nearly 3 years of pharmacy prescription information, but we did not assess for change in adherence over that time period, which may occur as patients live with and adapt to their medical conditions. All subjects included in the sample had at least 1 visit with a primary care specialty, which enhances the utility of these results to primary care practitioners, although prescriptions and some management may have been shared with specialists. Although the I2B2 system is a rich collection of important variables, some covariates, such as duration of diabetes, are not available. Future research should consider longitudinal studies to assess temporal trends in adherence and Alc variability.

We used pharmacy claims to measure medication adherence, which may overestimate adherence, since it cannot account for pill storing or pill dumping of acquired medications but has been shown to be a reliable and practical method for health services research. ${ }^{34}$ Future studies should consider replicating analyses using a more rigorous measure of adherence, such as pill counting or MEMS, to test the validity of these findings. We excluded subjects using insulin due to the complexities of measuring dose and compliance, the markedly increased Alc variability that insulin induces, and to achieve a more clinically homogenous sample of diabetic patients. If adherence to insulin could be efficiently measured, it would be important to test the utility of VVV of Alc in predicting adherence in that patient population. We did include subjects 
on relatively newer diabetic medications, such as incretin mimetics and DPP-4 inhibitors, while most previous studies on medication adherence among diabetics have been restricted to biguanides, sulfonylureas, or other oral agents. We could not account for medications that patients may have obtained without submitting pharmacy prescription claims data, such as free antidiabetic medications available at some large retailers. There is a risk of misclassification bias if patients were not continuously enrolled in the health plan during the study period. If patients temporarily disenrolled and received prescriptions through another plan and then re-enrolled in BMC Health Plan, their MPR would appear falsely low and may have biased our results.

Additional factors associated with poor medication adherence, including polypharmacy, ${ }^{12}$ dose frequency, ${ }^{35}$ burden of comorbid diseases, ${ }^{21}$ cost of copayments for medications,${ }^{36}$ and psychosocial factors such as personal health beliefs, ${ }^{37}$ health literacy, ${ }^{38}$ and depression, ${ }^{39}$ were not measured in this study. We did include specific diagnoses that often co-occur with diabetes, such as hypertension, or that might affect diabetic medication prescribing practices, such as chronic kidney disease. With the exception of hypertension, these diseases were not associated with nonadherence.

\section{Conclusions}

We found that VVV of Alc is not a robust indicator of nonadherence to noninsulin diabetic medications. Efficient, reliable means of detecting nonadherence at the bedside are still needed in order to diagnose at-risk patients and deliver intervention to appropriate patients. Researchers and health care systems should explore innovative approaches that leverage health information technology to measure adherence, such as integrating patient information (e.g., MEMS data) or pharmacy information directly into electronic health records to create useful alerts for physicians at the point of care.

\section{Authors}

AMBILI RAMACHANDRAN, MD, MS, is Assistant Professor of Medicine, Section of General Internal Medicine, and DEVIN M. MANN, MD, MS, is Assistant Professor of Medicine, Section of General Internal Medicine and Section of Preventive Medicine and Epidemiology, Department of Medicine, Boston University School of Medicine, Boston, Massachusetts. MICHAEL WINTER, MPH, is Associate Director, Data Coordinating Center, Boston University School of Public Health, Boston, Massachusetts.

AUTHOR CORRESPONDENCE: Ambili Ramachandran, MD, MS Assistant Professor of Medicine, General Internal Medicine, Boston University School of Medicine, 801 Massachusetts Ave., Crosstown Center, 2nd Fl., Boston, MA 02118. Tel.: 617.414.6955;

Fax: 617.414.4676; E-mail: ambili.ramachandran@bmc.org.

\section{DISCLOSURES}

This study was funded by Boston University's Clinical and Translational Institute (ULl-TR000157); American Cancer Society Physician in Training Award in Cancer Prevention (PTAPM-97-185-16); and Patient-Oriented Mentored Scientist Award through the National Institute of Diabetes, Digestive, and Kidney Diseases (K23DK081665). The funders had no involvement in the design and conduct of the study; collection, management, analysis, and interpretation of the data; or preparation, review, or approval of the manuscript. The authors declare no conflicts of interest.

Study concept and design were contributed by Mann, Winter, and Ramachandran. Winter collected data, assisted by Mann, and all authors contributed equally to data analysis. The writing and revision of the manuscript were carried out primarily by Ramachandran, assisted by Winter and Mann.

\section{REFERENCES}

1. Rubin RR. Adherence to pharmacologic therapy in patients with type 2 diabetes mellitus. Am J Med. 2005;118(Suppl 5A):S27-34.

2. Cramer JA. A systematic review of adherence with medications for diabetes. Diabetes Care. 2004;27(5):1218-24.

3. Adams AS, Trinacty CM, Zhang F, et al. Medication adherence and racial differences in Alc control. Diabetes Care. 2008;31(5):916-21.

4. Hertz RP, Unger A N, Lustik MB. Adherence with pharmacotherapy for type 2 diabetes: a retrospective cohort study of adults with employer-sponsored health insurance. Clin Ther. 2005;27(7):1064-73.

5. Yang Y, Thumula V, Pace PF, Banahan BF 3rd, Wilkin NE, Lobb WB Predictors of medication nonadherence among patients with diabetes in Medicare Part D programs: a retrospective cohort study. Clin Ther. 2009;31(10):2178-88

6. Zhu VJ, Tu W, Marrero DG, Rosenman MB, Overhage JM. Race and medication adherence and glycemic control: findings from an operational health information exchange. AMIA Annu Symp Proc. 2011;2011:1649-57.

7. Ho PM, Rumsfeld JS, Masoudi FA, et al. Effect of medication nonadherence on hospitalization and mortality among patients with diabetes mellitus. Arch Intern Med. 2006;166(17):1836-41.

8. Yu AP, Yu YF, Nichol MB. Estimating the effect of medication adherence on health outcomes among patients with type 2 diabetes - an application of marginal structural models. Value Health. 2010;13(8):1038-45.

9. Balkrishnan R, Rajagopalan R, Camacho FT, Huston SA, Murray FT, Anderson RT. Predictors of medication adherence and associated health care costs in an older population with type 2 diabetes mellitus: a longitudinal cohort study. Clin Ther. 2003;25(11):2958-71.

10. Egede LE, Gebregziabher M, Dismuke CE, et al. Medication nonadherence in diabetes: longitudinal effects on costs and potential cost savings from improvement. Diabetes Care. 2012;35(12):2533-39.

11. Miller LG, Liu H, Hays RD, et al. How well do clinicians estimate patients' adherence to combination antiretroviral therapy? J Gen Intern Med. 2002;17(1):1-11.

12. Osterberg L, Blaschke T. Adherence to medication. N Engl J Med. 2005;353(5):487-97.

13. Cramer JA, Mattson RH, Prevey ML, Scheyer RD, Ouellette VL. How often is medication taken as prescribed? A novel assessment technique. JAMA. 1989;261(22):3273-77.

14. Marcum ZA, Sevick MA, Handler SM. Medication nonadherence: a diagnosable and treatable medical condition. JAMA. 2013;309(20):2105-06.

15. Mann DM, Glazer NL, Winter M, et al. A pilot study identifying statin nonadherence with visit-to-visit variability of low-density lipoprotein cholesterol. Am J Cardiol. 2013;111(10):1437-42.

16. Muntner P, Levitan EB, Joyce C, et al. Association between antihypertensive medication adherence and visit-to-visit variability of blood pressure. J Clin Hypertens (Greenwich). 2013;15(2):112-17. 
17. Boston University Clinical and Translational Science Institute. Massachusetts Healthcare Disparities Repository (MHDR). Informatics for Integrating Biology \& the Bedside (i2b2). Available at: http://www.bu.edu/ i2b2/mhdr/. Accessed January 30, 2015.

18. Hess LM, Raebel MA, Conner DA, Malone DC. Measurement of adherence in pharmacy administrative databases: a proposal for standard definitions and preferred measures. Ann Pharmacother. 2006;40(7-8):1280-88.

19. Zhang Y, Baik SH, Chang CC, Kaplan CM, Lave JR. Disability, race/ethnicity, and medication adherence among Medicare myocardial infarction survivors. Am Heart J. 2012;164(3):425-433.e4.

20. Mann DM, Allegrante JP, Natarajan S, Halm EA, Charlson M. Predictors of adherence to statins for primary prevention. Cardiovasc Drugs Ther. 2007;21(4):311-16.

21. Mann DM, Woodward M, Muntner P, Falzon L, Kronish I. Predictors of nonadherence to statins: a systematic review and meta-analysis. Ann Pharmacother. 2010;44(9):1410-21.

22. Ismail-Beigi F. Clinical practice. Glycemic management of type 2 diabetes mellitus. N Engl J Med. 2012;366(14):1319-27.

23. Bersot T. Drug therapy for hypercholesterolemia and dyslipiedmia. In: Brunton L, Chabner B, Knollmann B, eds. Goodman and Gilman's The Pharmacological Basis of Therapeutics. 12th ed. New York: McGraw-Hill; 2011.

24. Gregg EW, Chen H, Wagenknecht LE, et al. Association of an intensive lifestyle intervention with remission of type 2 diabetes. JAMA. 2012;308(23):2489-96.

25. Knowler WC, Barrett-Connor E, Fowler SE, et al. Reduction in the incidence of type 2 diabetes with lifestyle intervention or metformin. N Engl J Med. 2002;346(6):393-403.

26. Herman WH, Ma Y, Uwaifo G, et al. Differences in AlC by race and ethnicity among patients with impaired glucose tolerance in the Diabetes Prevention Program. Diabetes Care. 2007;30(10):2453-57.

27. Ziemer DC, Kolm P, Weintraub WS, et al. Glucose-independent, blackwhite differences in hemoglobin Alc levels: a cross-sectional analysis of 2 studies. Ann Intern Med. 2010;152(12):770-77.

28. Wadén J, Forsblom C, Thorn LM, et al. Alc variability predicts incident cardiovascular events, microalbuminuria, and overt diabetic nephropathy in patients with type 1 diabetes. Diabetes. 2009;58(11):2649-55.
29. Sugawara A, Kawai K, Motohashi S, et al. $\mathrm{HbA}(1 \mathrm{c})$ variability and the development of microalbuminuria in type 2 diabetes: Tsukuba Kawai Diabetes Registry 2. Diabetologia. 2012;55(8):2128-31.

30. Rodríguez-Segade S, Rodríguez J, García López JM, Casanueva FF, Camiña F. Intrapersonal $\mathrm{HbA}(\mathrm{lc})$ variability and the risk of progression of nephropathy in patients with Type 2 diabetes. Diabet Med. 2012;29(12):1562-66.

31. Ma WY, Li HY, Pei D, et al. Variability in hemoglobin Alc predicts allcause mortality in patients with type 2 diabetes. J Diabetes Complications. 2012;26(4):296-300.

32. Rothwell PM, Howard SC, Dolan E, et al. Prognostic significance of visitto-visit variability, maximum systolic blood pressure, and episodic hypertension. Lancet. 2010;375(9718):895-905.

33. Muntner P, Shimbo D, Tonelli M, Reynolds K, Arnett DK, Oparil S. The relationship between visit-to-visit variability in systolic blood pressure and all-cause mortality in the general population: findings from NHANES III, 1988 to 1994. Hypertension. 2011;57(2):160-66.

34. Andrade SE, Kahler KH, Frech F, Chan KA. Methods for evaluation of medication adherence and persistence using automated databases. Pharmacoepidemiol Drug Saf. 2006;15(8):565-74.

35. Claxton AJ, Cramer J, Pierce C. A systematic review of the associations between dose regimens and medication compliance. Clin Ther. 2001;23(8):1296-310.

36. Piette JD, Heisler M, Wagner TH. Problems paying out-of-pocket medication costs among older adults with diabetes. Diabetes Care. 2004;27(2):384-91.

37. Glasgow RE, Hampson SE, Strycker LA, Ruggiero L. Personal-model beliefs and social-environmental barriers related to diabetes self-management. Diabetes Care. 1997;20(4):556-61.

38. Schillinger D, Grumbach K, Piette J, et al. Association of health literacy with diabetes outcomes. JAMA. 2002;288(4):475-82.

39. Lin EH, Katon W, Von Korff M, et al. Relationship of depression and diabetes self-care, medication adherence, and preventive care. Diabetes Care. 2004;27(9):2154-60. 